\title{
Si Incorporation in InP Nanowires Grown by Au-Assisted Molecular Beam Epitaxy
}

\author{
Lorenzo Rigutti, ${ }^{1}$ Andres De Luna Bugallo, ${ }^{1}$ Maria Tchernycheva, ${ }^{1}$ Gwenole Jacopin, ${ }^{1}$ \\ François H. Julien, ${ }^{1}$ George Cirlin, ${ }^{2,3}$ Gilles Patriarche, ${ }^{2}$ Damien Lucot, ${ }^{2}$ \\ Laurent Travers, ${ }^{2}$ and Jean-Christophe Harmand ${ }^{2}$ \\ ${ }^{1}$ Institut d'Electronique Fondamentale, UMR 8622 CNRS, Université Paris Sud XI, 91405 Orsay, France \\ ${ }^{2}$ Laboratoire de Photonique et Nanostructures, UPR 20 CNRS, Route de Nozay, 91460 Marcoussis, France \\ ${ }^{3}$ St Petersburg Physics and Technology Center for Research and Education RAS, Khlopina 8/3, St Petersburg 195220, Russia
}

Correspondence should be addressed to Maria Tchernycheva, maria.tchernycheva@ief.u-psud.fr

Received 9 July 2009; Revised 26 October 2009; Accepted 19 November 2009

Recommended by Sanjay Mathur

We report on the growth, structural characterization, and conductivity studies of Si-doped InP nanowires grown by Au-assisted molecular beam epitaxy. It is shown that Si doping reduces the mean diffusion length of adatoms on the lateral nanowire surface and consequently reduces the nanowire growth rate and promotes lateral growth. A resistivity as low as $5.1 \pm 0.3 \times 10^{-5} \Omega \cdot \mathrm{cm}$ is measured for highly doped nanowires. Two dopant incorporation mechanisms are discussed: incorporation via catalyst particle and direct incorporation on the nanowire sidewalls. The first mechanism is shown to be less efficient than the second one, resulting in inhomogeneous radial dopant distribution.

Copyright ( 92009 Lorenzo Rigutti et al. This is an open access article distributed under the Creative Commons Attribution License, which permits unrestricted use, distribution, and reproduction in any medium, provided the original work is properly cited.

\section{Introduction}

Free-standing semiconductor nanowires (NWs) attract an increasing attention due to their emerging applications to nanoscale optoelectronic devices [1]. Big effort has been devoted to the fabrication of $\operatorname{In}(\mathrm{As}) \mathrm{P}$ NWs and InAsP/InP heterostructures for near infrared light emission/detection applications [2-6]. InP NWs have been synthesized by different epitaxial techniques including laser-assisted catalytic growth [2], metal-organic vapor phase epitaxy (MOVPE) [7-9], chemical beam epitaxy [10], and molecular beam epitaxy (MBE) [11]. InP NWs containing p-n junction have been grown by laser-assisted catalytic growth and MOVPE $[12,13]$.

The fabrication of optoelectronic devices requires accurate control of NW doping. To control the doping, it is necessary to understand how, under which conditions, and in which quantities dopants are incorporated into NWs. The description of the dopant incorporation by the catalystassisted growth is not straightforward. Indeed, two mechanisms of impurity incorporation in NWs are possible- the incorporation via vapor-liquid-solid (VLS) mechanism from the catalyst and the direct incorporation on the NW sidewalls via lateral growth. Another important issue is the influence of the doping adatom on the NW growth itself and the possible changes of morphology and crystalline structure. These issues have been partly addressed for catalyst-assisted MOVPE growth of InP NWs [13]. It has been shown that zinc incorporation in InP has a major effect changing the crystalline structure from wurtzite to zinc-blende [14]. The effect of doping on the emission properties of InP NWs grown by laser ablation was described in [15]. To the best of our knowledge, no study of doping issues has been carried out for MBE grown InP NWs.

In the present work we investigate the growth and conductivity of Au-assisted MBE grown InP NWs doped with $\mathrm{Si}$ at different concentrations. We analyze the morphological changes induced by Si incorporation and its effect on the NW resistivity. We show that Si flux reduces the adatom mobility and promotes the lateral growth. Structural characterization shows that the NW crystalline structure is not affected by $\mathrm{Si}$ incorporation: it remains wurtzite with some cubic 
inclusions as in the case of undoped InP NWs. The doping incorporation in the NW core via VLS mechanism is shown to be less efficient than the incorporation in the NW shell via lateral growth.

\section{InP Nanowire Growth}

The InP NWs were grown on InP (111)B substrates in a solid-source MBE system using Au as a catalyst for the NW growth. Prior to the NW growth, the substrate surface was deoxidized at $530^{\circ} \mathrm{C}$ and a $100 \mathrm{~nm}$ thick InP buffer layer was grown. Gold was deposited under phosphorus flux at $360^{\circ} \mathrm{C}$ using an Au effusion cell installed in the III-V growth chamber. The gold amount is estimated to be equivalent to $1 \mathrm{~nm}$ layer. For all samples the nominal growth rate, that is, the growth rate on a clean and $\mathrm{Au}$-free InP surface, was fixed at $12 \mathrm{~nm} / \mathrm{min}$.

The NW growth was performed in two steps: during the first 5 minutes the substrate temperature was kept constant at $360^{\circ} \mathrm{C}$ and then it was increased during approximately 1 minute to $410^{\circ} \mathrm{C}$ and kept at this value for 20 minutes. This two-step procedure was used to produce dense InP NW ensembles with almost cylindrical shape. Indeed, the low temperature of $360^{\circ} \mathrm{C}$ is favorable to initiate the NW formation, but the growth rate is rather low $(54 \mathrm{~nm} / \mathrm{min})$ and a nucleation on the NW sidewalls takes place. At $410^{\circ} \mathrm{C}$ the lateral growth is minimized and the growth rate is increased, because the high temperature increases the mean diffusion length of adatoms. The temperature domain for InP NW formation and the change of the NW morphology with growth temperature are described in more detail in $[11,16]$. One disadvantage of the high growth temperature is that the NW formation is partly suppressed, resulting in a low density of NWs. The reduction of the NW density at high growth temperatures has also been reported for InAs MBE-grown NWs [17]. It was suggested that at high temperature it is more difficult for the catalyst particle to collect enough In and to increase its size above the GibbsThomson critical diameter. So many catalyst particles remain on the substrate surface without NW formation [17]. This is in agreement with our observations for InP NWs. However, we observed that if the initial NW formation step is done at low temperature, the NW growth continued at $410^{\circ} \mathrm{C}$ and the NWs acquire cylindrical shape. We used the two-temperature procedure to grow InP NWs of this study.

To test the effect of Si doping, a series of 5 samples was grown with different $\mathrm{Si}$ concentrations. Si doping was only introduced during the last 20 minutes of the growth. The first sample is nominally undoped, the other four samples were grown with $\mathrm{Si}$ cell temperature maintained at $1040^{\circ} \mathrm{C}, 1070^{\circ} \mathrm{C}, 1100^{\circ} \mathrm{C}$, and $1150^{\circ} \mathrm{C}$, respectively. The equivalent $2 \mathrm{D}$ doping is in the $3 \times 10^{17}-4 \times 10^{18} \mathrm{~cm}^{-3}$ range, the exact values are given in Table 1 . It was calibrated by Hall measurements on two-dimensional GaAs(111)B layers deposited at $12 \mathrm{~nm} / \mathrm{min}$ growth rate.

The resulting morphology of NW ensembles was investigated by scanning electron microscopy (SEM). In the undoped sample the NWs have almost cylindrical shape
TABLE 1: NW growth rate for different Si doping levels.

\begin{tabular}{lccc}
\hline $\mathrm{T}_{\mathrm{Si}}\left({ }^{\circ} \mathrm{C}\right)$ & $\begin{array}{c}\text { Equivalent 2D } \\
\text { doping }\left(\mathrm{cm}^{-3}\right)\end{array}$ & $\begin{array}{c}\text { NW average } \\
\text { length }(\mu \mathrm{m})\end{array}$ & $\begin{array}{c}\text { NW growth } \\
\text { rate }(\mathrm{nm} / \mathrm{min})\end{array}$ \\
\hline closed & undoped & 2.7 & 119 \\
1040 & $3 \times 10^{17}$ & 2.57 & 112 \\
1070 & $5 \times 10^{17}$ & 2.53 & 110 \\
1100 & $1.1 \times 10^{18}$ & 2.53 & 110 \\
1150 & $3.7 \times 10^{18}$ & 1.2 & 44 \\
\hline
\end{tabular}

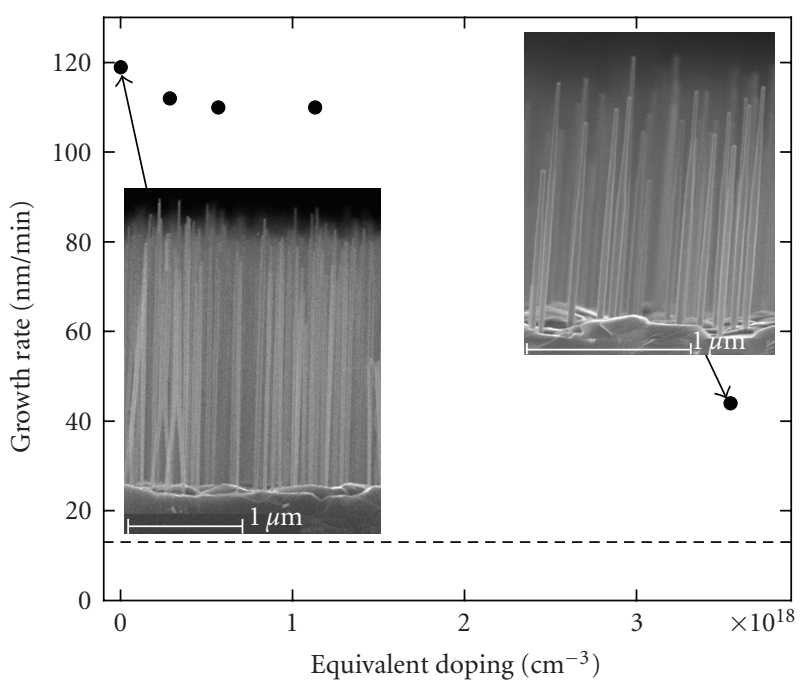

FIGURE 1: NW growth rate as a function of the equivalent Si doping level. Dashed line shows the nominal deposition rate. Insets show SEM images of undoped (left) and heavily doped (right) InP NWs.

with diameter at the base of $25-30 \mathrm{~nm}$ and the NW length of $2.7 \pm 0.2 \mu \mathrm{m}$ (cf. inset to Figure 1). The growth rate at $360^{\circ} \mathrm{C}$ was calibrated separately and is equal $54 \mathrm{~nm} / \mathrm{min}$. Using this value for the first 6 minutes of deposition, we calculate the growth rate for undoped NWs at $410^{\circ} \mathrm{C}$ equal to $119 \mathrm{~nm} / \mathrm{min}$.

At low Si fluxes (equivalent doping $3 \times 10^{17}-1 \times$ $10^{18} \mathrm{~cm}^{-3}$ ), the NWs acquire a tapered shape towards the catalyst. The diameter in the lower cylindrical part is increased to $45-55 \mathrm{~nm}$. In this doping range, Si incorporation reduces slightly the NW growth rate to $\sim 110 \mathrm{~nm} / \mathrm{min}$. The change of NW morphology indicates that when the Si flux is introduced, nucleation on the lateral facets takes place in addition to the vertical growth. This phenomenon occurs when the mean diffusion length of species $\left\langle\lambda_{\text {diff }}\right\rangle$ on the lateral NW facets becomes comparable or smaller than the NW height. In this case, most of species supplied by the surface where the NWs stand cannot reach the catalyst droplets and nucleate on the NW sidewalls. The growth on the sidewalls may occur in a layer by layer fashion to minimize the steps at the sidewall surfaces. This explains why along most of their length, the NWs have a constant diameter. At the top the wires develop tapering to recover the smaller diameter of the catalyst particle. 


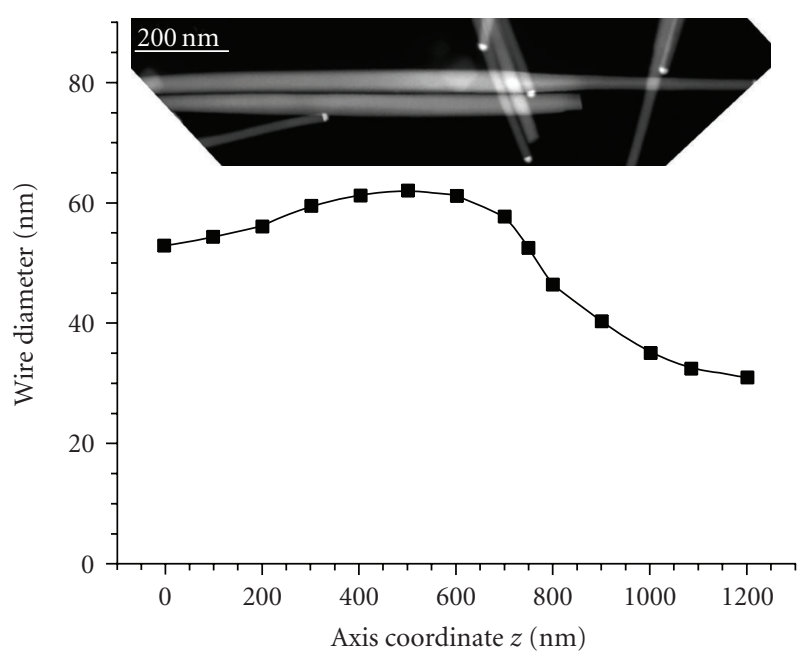

Figure 2: Variation of the NW diameter along the NW growth direction. Inset shows a HAADF STEM image of highly doped NWs. The diameter was measured for the upper horizontal NW of the image.

When the Si flux is further increased (equivalent doping $3.7 \times 10^{18} \mathrm{~cm}^{-3}$ ), the NW growth rate dramatically decreases to $44 \mathrm{~nm} / \mathrm{min}$. The $\mathrm{NW}$ diameter $(40-50 \mathrm{~nm})$ remains superior to the one of undoped NWs, but is slightly smaller than for NWs with low Si doping. The total amount of material in NWs is also reduced. The reduction of the growth rate and the NW morphology change were confirmed by a fabrication of a second identical sample, which excludes any growth-related accidents. These results can be explained by the further reduction of the mean diffusion length of species for higher Si fluxes. If $\left\langle\lambda_{\text {diff }}\right\rangle$ becomes much smaller than the NW length, the layer-by-layer growth on the entire NW facet is no longer possible. Moreover, the axial growth via catalyst particle cannot be supplied by the species diffusing from the substrate. To understand where species come from, one should consider the typical design of the MBE machines for which the molecular beams arrive with an incident angle about $30^{\circ}$ with respect to the perpendicular direction to the substrate. It means that a significant part of flux directly impinges the NW sidewalls. This provides adatoms for the axial and lateral growth, even when the NW length is above $\left\langle\lambda_{\text {diff }}\right\rangle$. However, the adatom quantity is smaller, which explains the reduction in the growth rate.

It can be seen in the SEM images of Figure 1 that for high Si doping the NW lower part is no longer cylindrical, but acquires a "barrel" shape: the NW diameter is maximal in the middle part of the NW and decreases towards the NW bottom. This particular shape was also observed by scanning transmission electron microscopy. Figure 2 illustrates the variation of the diameter measured along the axis of the NW whose high angle annular dark field (HAADF) STEM image is reported in the inset. The diameter increases by $\sim 10 \mathrm{~nm}$ for the first $500 \mathrm{~nm}$ and then decreases by $\sim 30 \mathrm{~nm}$ towards the catalyst.

This unusual shape can be explained by the shadowing effect during the growth. Indeed, for an ensemble of NWs of large enough length, the species impinge mostly the upper part of the NW lateral surface because the lower part of the NW is shadowed by the neighboring NWs. The small mean diffusion length prevents the adatom migration on the NW surface. As a consequence, the lower NW part is poorly supplied in adatoms and has a small lateral growth rate. The diameter reduction towards the NW top is due to the species consumption in the catalyst by the axial NW growth.

\section{Structural Analyses}

The crystalline structure of Si doped InP NWs was studied by means of transmission electron microscopy (TEM) and scanning transmission electron microscopy (STEM). The microscope is equipped with an energy-dispersive X-ray (EDX) spectrometer for composition measurements. For TEM characterizations the NWs were mechanically detached from their substrate and picked up by touching the substrate with a TEM grid.

Figure 3(a) displays a high-resolution TEM image of two InP NWs highly doped with Si (equivalent doping $3.7 \times$ $10^{18} \mathrm{~cm}^{-3}$ ). The crystalline structure is wurtzite. The only defects observed in NWs are stacking faults perpendicular to the NW axis. The stacking faults correspond to several (up to 5) planes with cubic atomic arrangement inserted in a wurtzite structure. The average density of these defects is about 1 per $50 \mathrm{~nm}$ of NW length; however defect-free regions as long as $200 \mathrm{~nm}$ can be found. It should be noted that the stacking faults represent a typical defect observed in MBE-grown InP [11], InAs [17] or GaAs [18], NWs; their appearance is not related to $\mathrm{Si}$ doping. No specific modifications of the NW crystalline structure related to $\mathrm{Si}$ incorporation could be identified.

Figures 3(b) and 3(c) show the high magnification STEM images with bright field and HAADF observation conditions, respectively. STEM observations confirm the presence of cubic insertions in NWs (the position of 5 consecutive cubic planes in the images (b) and (c) is marked with arrows). No Si-rich regions could be identified by STEM, which excludes Si segregation in the NW body or on the surface.

The EDX spectroscopy was used to analyze the composition of the NWs and catalyst particles. No presence of Si could be identified in the NW body down to the EDX detection limit $(\sim 0.3$ atomic percent). This is in agreement with the expected doping concentration below $1 \times 10^{19} \mathrm{~cm}^{-3}$, which is too low to be detected by EDX technique. However, the presence of $\sim 1$ atomic percent of $\mathrm{Si}$ in the catalyst particle was observed. The catalyst particle mainly contains $\mathrm{Au}$ and In, the measured alloy composition is close to the $A_{0.5} \operatorname{In}_{0.5}$ stable phase [19].

\section{Conductivity Study of n-Doped InP Nanowires}

For conductivity study the NWs were cut by ultra-sonic ethanol bath and dispersed on a $\mathrm{SiO}_{2} / \mathrm{Si}(001)$ template with alignment marks. A $150 \mathrm{~nm}$ thick $\mathrm{SiO}_{2}$ insulating layer was prepared by dry oxidation and the alignment marks 


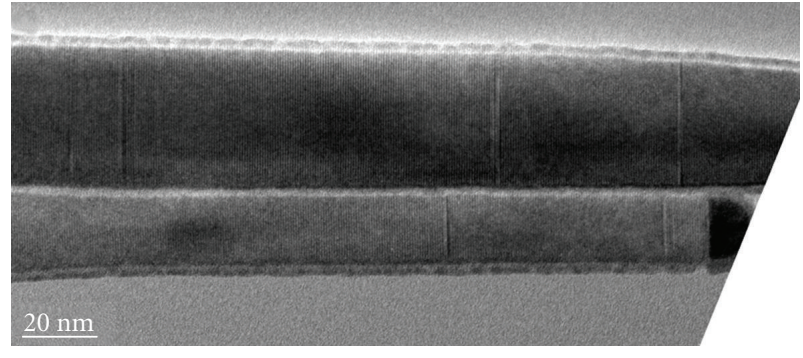

(a)

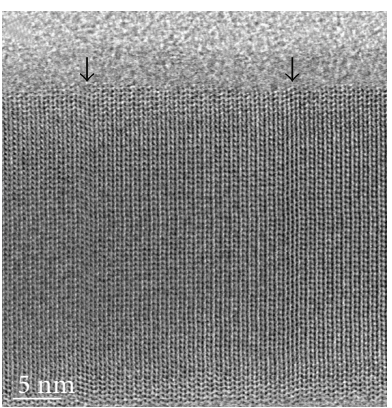

(b)

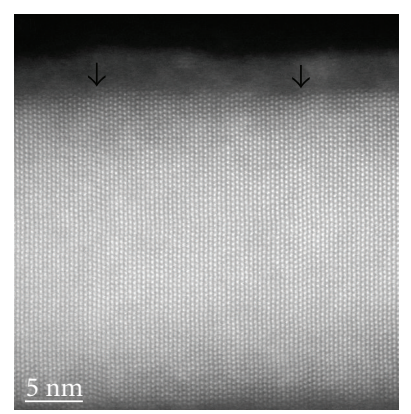

(c)
Figure 3: (a) TEM image for $\langle 1-210\rangle$ zone axis of Si-doped InP NWs; (b) high magnification bright field STEM image; (c) STEM HAADF image of the same zone as (b). Black arrows in (b) and (c) indicate the position of stacking faults.

were fabricated using e-beam lithography, metallization, and lift-off procedure. Several NWs were chosen, their position with respect to the alignment marks was located using SEM microscope and the mask for contact deposition was designed. After electron lithography and development, the sample was cleaned in diluted $\mathrm{HCl}$ to deoxidize the $\mathrm{NW}$ surface and $\mathrm{Ni}(5 \mathrm{~nm}) / \mathrm{Ge}(30 \mathrm{~nm}) / \mathrm{Au}(60 \mathrm{~nm}) / \mathrm{Ni}$ $(30 \mathrm{~nm}) / \mathrm{Au}(100 \mathrm{~nm})$ metallization was deposited. To reduce the contact resistance, samples were annealed for 1 minute at $380^{\circ} \mathrm{C}[20,21]$.

Some authors claim that the electron beam lithography is not well suited for NW contacting because it may induce some irreversible surface contamination by the e-beam resist [22]. Indeed, NW conductivity is sensitive to the ambient conditions and to any surface adsorbates especially in the case of thin NWs [23]. The alternative fabrication techniques for NW contacting such as metal deposition by focused ion beam (FIB) $[24,25]$ or shadow mask metal deposition [26] have been proposed. The major advantages of electron lithography and metal lift-off contacting technique are the ultimate alignment precision (below $10 \mathrm{~nm}$ ), which is crucial for nanowire structures with length $l<5 \mu \mathrm{m}$, the low induced damage and the possibility to deposit different metal sequences. The detrimental influence of residual organic contamination can be minimized by plasma cleaning.

The I-V measurements were performed on the following 3 NW samples: undoped NWs, NWs with low doping level (equivalent to $3 \times 10^{17} \mathrm{~cm}^{-3}$ ), and NWs with high doping level (equivalent to $3.7 \times 10^{18} \mathrm{~cm}^{-3}$ ). About $10 \mathrm{NWs}$ were analyzed for each sample. The conduction was probed at

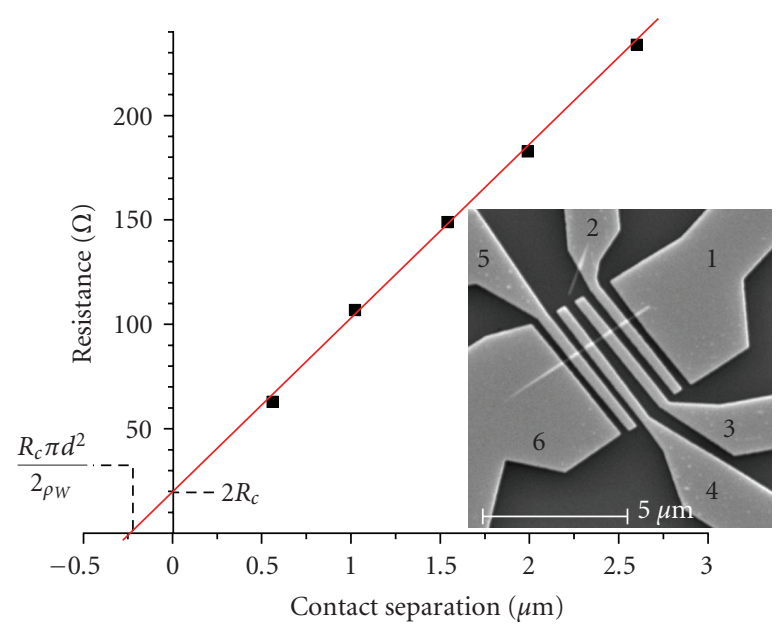

FIGURE 4: TLM analysis of the NW resistivity. Inset shows SEM image of InP NW with 6 contacts (contact 1 corresponds to the NW bottom and contact 6-to the NW conical top).

room temperature under ambient pressure using a needle probe station and a Keithley 2636 source meter. For undoped NWs no conduction was observed within the experimental accuracy down to $0.1 \mathrm{pA}$. This observation is strongly different from the results for MBE-grown InAs NWs who are shown to be conductive and form ohmic contacts with no intentional doping [27].

Si-doped InP NWs were found to be conductive with slightly Schottky behavior before annealing and ohmic behavior afterwards. The resistance between 2 contacts in the central NW part separated by $0.5 \mu \mathrm{m}$ distance is $1540 \Omega$ $(60 \Omega)$ for NWs with equivalent doping of $3 \times 10^{17} \mathrm{~cm}^{-3}$ $\left(3.7 \times 10^{18} \mathrm{~cm}^{-3}\right)$, respectively.

To extract independently the NW resistance and the contact resistance, we have applied to NWs the transmission line model (TLM) widely used for thin film semiconductors [28] and applied also in the case of semiconductor nanowires [29]. Additional samples were grown with $6 \mu \mathrm{m}$ long Sidoped InP NWs. The NWs were processed with 6 contacts as illustrated in the inset to Figure 4 (contact 1 corresponds to the NW bottom and contact 6-to the NW conical top).

We analyzed the resistance between the contact pairs 1-2, $1-3,1-4,1-5$, and 1-6 as a function of the contact separation. The analysis showed that the resistance is uniformly distributed along the lower cylindrical part of the NW (contacts 1 to 5 ). In contrast, for the resistance between contacts 1 and 6 we found strong fluctuations from wire to wire depending on the NW portion covered with the contact. For the NW shown in the inset to Figure 2, contact 6 covers entirely the conical part and partly the cylindrical part. For this NW, the resistance between contacts 1 and 6 scales linearly with the contact separation. However for other NWs from the same growth run for which the contact 6 was deposited only on the conical part, the resistance between contacts 1 and 6 was strongly increased (by a factor of 3-5). This increase cannot be simply explained by geometrical parameters as will be discussed in the next section. 
For highly doped NWs (the equivalent doping of $3.7 \times$ $10^{18} \mathrm{~cm}^{-3}$ ) the TLM analysis of contacts 1 to 5 yields a contact resistance equal to $R_{c}=10 \pm 2 \Omega$ (determined from the intersection of the linear fit with the ordinate axis) and a wire resistance for unit length $r_{w}=80 \pm$ $4 \Omega \mu \mathrm{m}^{-1}$ (determined from the intersection of the linear fit with the abscissa axis). With a diameter value $d_{N W} \sim$ $90 \mathrm{~nm}$ one obtains the resistivity value $\rho_{W}=5.1 \pm$ $0.3 \times 10^{-5} \Omega \mathrm{cm}$ (under the hypothesis of a homogeneous conduction through the wire cross section).

TLM analysis on weakly doped NWs (the equivalent doping of $3 \times 10^{17} \mathrm{~cm}^{-3}$ ) showed higher dispersion with the average values for the contact resistance $R_{c}=400 \pm 200 \Omega$, wire resistance for unit length $r_{w}=740 \pm 160 \Omega \mu \mathrm{m}^{-1}$, and resistivity value of $\rho_{W}=1.9 \pm 0.4 \times 10^{-4} \Omega \mathrm{cm}$.

\section{Inhomogeneous Doping Incorporation in Nanowires}

We observed in both weakly doped and strongly doped NWs that the resistance measured in the top part of the NW was systematically higher than the resistance measured in the central or bottom parts. This effect is stronger than what is expected from the NW cross-section reduction in the top part and indicates a nonuniform distribution of dopants along the NW axis. Indeed, two mechanisms of dopant incorporation in NWs are possible-the incorporation via VLS growth from the catalyst and the direct incorporation on the NW sidewalls via lateral growth. In the cylindrical part of the NW the contacts are deposited on the InP shell formed by lateral growth whereas in the top conical part of the NW the lateral growth layer is much thinner and vanishes close to the catalyst so that the top contact mainly probes the conduction through the NW core.

The Si incorporation into NWs by a VLS process could not be assessed by a direct measurement. However, the presence of $\mathrm{Si}$ in the catalyst particle revealed by EDX analyses suggests that this incorporation takes place. To demonstrate that the doping can also be incorporated by nucleation on the NW sidewalls, we grew a sample containing undoped/ndoped homojunction. The growth follows the previously described two step procedure with the first 10 minutes of high temperature growth with no intentional doping and the last 10 minutes with high Si doping. The SEM analyses of the NW shape show that the diameter close to the NW top is about $25-30 \mathrm{~nm}$ but is increased by the lateral growth to 50-60 nm in the lower NW part (Figure 5(a)). We deposited 4 contacts on the NWs so that the lower contacts (1 and 2) probe the nominally undoped NW part and the upper contacts ( 3 and 4 ) probe the doped part. The measurements were repeated for $5 \mathrm{NWs}$ from the same sample, with no substantial differences between them.

Figure 5(b) displays the $I-V$ curves measured for different contact combinations in one of the 5 analyzed NWs. The measurements show that the nominally undoped NW part is conductive; the resistance between contacts 1 and 2 is as low as $220 \Omega$. This demonstrates that $\mathrm{Si}$ doping can be efficiently incorporated via the lateral growth of InP shell.
The resistance is slightly higher than the one measured on the homogeneously highly doped NWs $(60 \Omega)$. This should be due to the presence of the undoped core in the undoped/ndoped homojunction NWs.

As seen in Figure 5, the resistance dramatically increases for the contact number 4 : it is about $2100 \Omega$ for contact couples 1-4, 2-4, and 3-4. According to the equivalent electrical scheme shown in Figure 5(c), the following system of linear equations is deduced from the $I-V$ curves:

$$
\begin{gathered}
R_{1}+r_{12}+r_{23}+r_{34}+R_{4}=2.6 \mathrm{k} \Omega, \\
R_{1}+r_{12}+r_{23}+R_{3}=0.52 \mathrm{k} \Omega, \\
R_{1}+r_{12}+R_{2}=0.22 \mathrm{k} \Omega, \\
R_{2}+r_{23}+r_{34}+R_{4}=2.5 \mathrm{k} \Omega, \\
R_{2}+r_{23}+R_{3}=0.38 \mathrm{k} \Omega, \\
R_{3}+r_{34}+R_{4}=2.4 \mathrm{k} \Omega,
\end{gathered}
$$

where $R_{i}$ is the resistance of contact $i$ and $r_{i j}$ is the resistance of the NW portion between contacts $i$ and $j$. The general solution is given by

$$
\begin{gathered}
R_{1}+r_{12}=0.17 \mathrm{k} \Omega, \\
R_{2}=0.05 \mathrm{k} \Omega, \\
r_{23}=0.19 \mathrm{k} \Omega, \\
R_{3}=0.15 \mathrm{k} \Omega, \\
R_{4}+r_{34}=2.25 \mathrm{k} \Omega .
\end{gathered}
$$

As seen, $R_{1}\left(R_{4}\right)$ and $r_{12}\left(r_{34}\right)$ cannot be determined independently without additional assumptions. Nevertheless, the sum of resistances $R_{4}+r_{34}$ is much higher than for other contacts. This cannot be simply explained by geometrical considerations: the NW diameter is reduced only by a factor of 1.75 and the surface of the contact 4 is almost equal to that of contacts 2 and 3 (it has smaller diameter but larger length). It means that in the top conical part the NW resistivity and/or the specific contact resistivity are affected. This could be due to the lower carrier concentration in the top part of the NW which means that the doping is less efficient in the core than in the shell.

The NW resistivity is estimated in the assumption that the contact resistance $R_{4}\left(R_{1}\right)$ is close to $R_{3}\left(R_{2}\right)$, respectively, and taking into account the NW diameter between the contacts measured from SEM images. The resistivity values are summarized in Table 2. The error margin refers to the measurement on the individual NW presented in Figure 5 and is determined by the uncertainties in the geometrical SEM measurements and in the electrical measurements. The resistivities of the different portions of the wire found in the other analyzed NWs are consistent within the error with those presented in Table 2.

As seen from Table 2, the NW resistivity increases towards the top. In the upper conical part it is 7 times larger than in the bottom cylindrical part and almost 12 times 


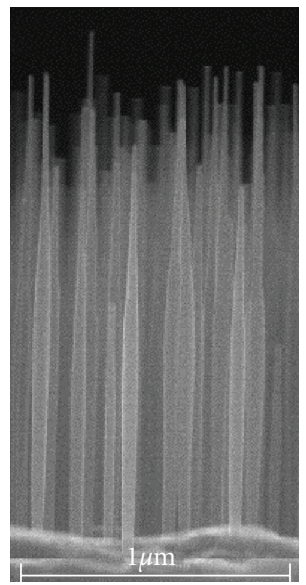

(a)

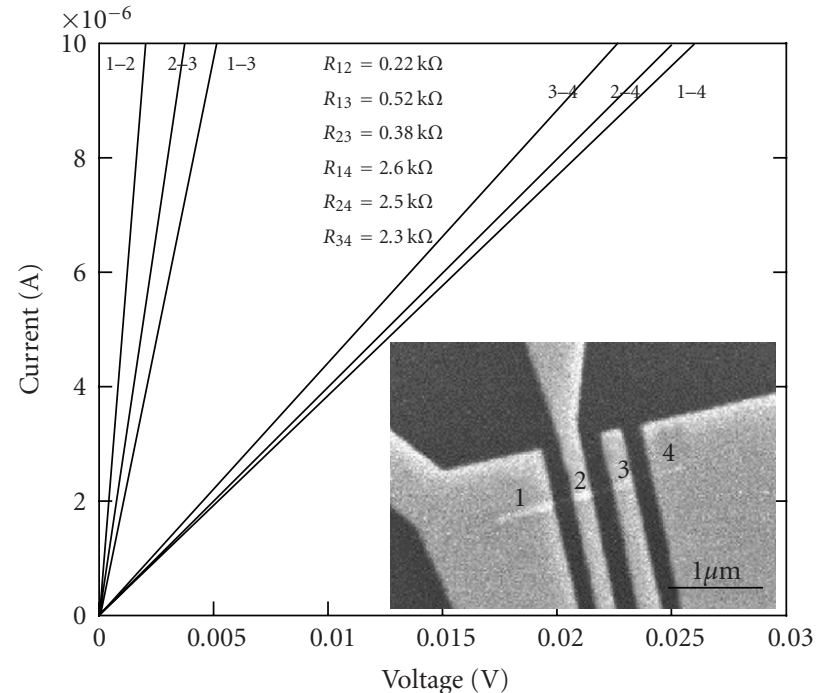

(b)

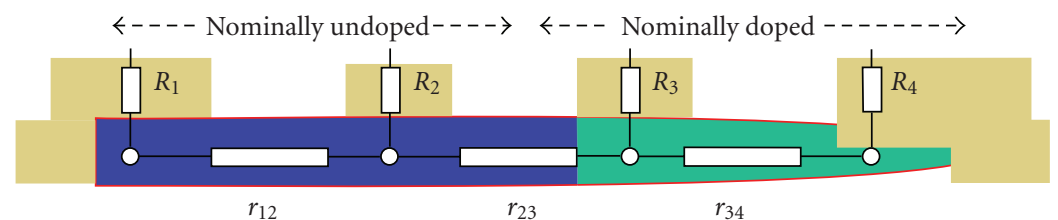

(c)

FIGURE 5: (a) SEM image of InP NWs containing undoped/n-doped homojunction. (b) $I-V$ curves between different contacts, the corresponding resistances are indicated in the plot. Inset shows an SEM image of a contacted homojunction NW (contact 4 corresponds to the NW top), (c) electrical scheme.

TABLE 2: Resistivity of inhomogeneously doped NWs.

\begin{tabular}{lccccc}
\hline Contacts $i-j$ & Diameter $(\mathrm{nm})$ & Separation $(\mathrm{nm})$ & Resistance $r_{i j}(\Omega)$ & Resistivity $(\Omega \mathrm{cm})$ & Error margin \\
\hline $1-2$ & 70 & 460 & 110 & $9.5 \times 10^{-5}$ & $\sim 20 \%$ \\
$2-3$ & 60 & 510 & 200 & $1.2 \times 10^{-4}$ & $\sim 20 \%$ \\
$3-4$ & 40 & 425 & 2100 & $6.3 \times 10^{-4}$ & $\sim 20 \%$ \\
\hline
\end{tabular}

larger than the value deduced for homogeneously highly doped NWs $\left(5.1 \times 10^{-5} \Omega \mathrm{cm}\right)$. This demonstrates that the dopant incorporation is much more efficient in the shell via lateral growth than in the core via VLS mechanism. The factor of 12 on the resistivity could be underestimated. Indeed, it is difficult to extract the exact value of the NW core conduction, because in the tapered part we are probing the conduction of both the core and the shell.

\section{Conclusions}

In conclusion, we have studied $\mathrm{Si}$ incorporation in InP nanowires grown by Au-assisted MBE. We have analyzed the morphological changes induced by $\mathrm{Si}$ incorporation at different concentrations and the effect on the NW resistivity. Si doping favors lateral growth and reduces the axial growth rate. Based on the morphology change induced by $\mathrm{Si}$ introduction we conclude that the presence of $\mathrm{Si}$ reduces the adatom diffusion length on the nanowire sidewalls. By measuring the resistivity of different nanowire parts we have shown that the doping incorporation in the NW core via catalyst is less efficient than the incorporation in the NW shell via lateral growth.

\section{Acknowledgment}

The financial support of the Project ANR-08-NANO-031 "BoNaFo" is acknowledged.

\section{References}

[1] Y. Li, F. Qian, J. Xiang, and C. M. Lieber, "Nanowire electronic and optoelectronic devices," Materials Today, vol. 9, no. 10, pp. 18-27, 2006.

[2] X. Duan, Y. Huang, Y. Cui, J. Wang, and C. M. Lieber, "Indium phosphide nanowires as building blocks for nanoscale electronic and optoelectronic devices," Nature, vol. 409, no. 6816, pp. 66-69, 2001.

[3] H. Pettersson, J. Trägårdh, A. I. Persson, L. Landln, D. Hessman, and L. Samuelson, "Infrared photodetectors in 
heterostructure nanowires," Nano Letters, vol. 6, no. 2, pp. 229-232, 2006.

[4] E. D. Minot, F. Kelkensberg, M. van Kouwen, et al., "Single quantum dot nanowire LEDs," Nano Letters, vol. 7, no. 2, pp. 367-371, 2007.

[5] A. Maharjan, K. Pemasiri, P. Kumar, et al., "Room temperature photocurrent spectroscopy of single zincblende and wurtzite InP nanowires," Applied Physics Letters, vol. 94, no. 19, Article ID 193115, 2009.

[6] J. Trägårdh, A. I. Persson, J. B. Wagner, D. Hessman, and L. Samuelson, "Measurements of the band gap of wurtzite In $A s_{1-x} P_{x}$ nanowires using photocurrent spectroscopy," Journal of Applied Physics, vol. 101, no. 12, Article ID 123701, 2007.

[7] S. Bhunia, T. Kawamura, Y. Watanabe, S. Fujikawa, and K. Tokushima, "Metalorganic vapor-phase epitaxial growth and characterization of vertical InP nanowires," Applied Physics Letters, vol. 83, no. 16, pp. 3371-3373, 2003.

[8] U. Krishnamachari, M. Borgstrom, B. J. Ohlsson, et al., "Defect-free InP nanowires grown in [001] direction on InP (001)," Applied Physics Letters, vol. 85, no. 11, pp. 2077-2079, 2004.

[9] H. Goto, K. Nosaki, K. Tomioka, S. Hara, K. Hiruma, J. Motohisa, and T. Fukui, "Growth of core-shell InP nanowires for photovoltaic application by selective-area metal organic vapor phase epitaxy," Applied Physics Express, vol. 2, no. 3, Article ID 035004, 2009.

[10] G. Radhakrishnana, A. Freundlicha, and B. Fuhrmann, "Chemical beam epitaxy of highly ordered network of tilted InP nanowires on silicon," Journal of Crystal Growth, vol. 311, no. 7, pp. 1855-1858, 2009.

[11] M. Tchernycheva, G. E. Cirlin, G. Patriarche, et al., "Growth and characterization of InP nanowires with InAsP insertions," Nano Letters, vol. 7, no. 6, pp. 1500-1504, 2007.

[12] M. S. Gudiksen, L. J. Lauhon, J. Wang, D. C. Smith, and C. M. Lieber, "Growth of nanowire superlattice structures for nanoscale photonics and electronics," Nature, vol. 415, no. 6872, pp. 617-620, 2002.

[13] M. T. Borgström, E. Norberg, P. Wickert, et al., "Precursor evaluation for in situ InP nanowire doping," Nanotechnology, vol. 19, no. 44, Article ID 445602, p. 6, 2008.

[14] R. E. Algra, M. A. Verheijen, M. T. Borgström, et al., "Twinning superlattices in indium phosphide nanowires," Nature, vol. 456, no. 7220, pp. 369-372, 2008.

[15] M. H. M. van Weert, O. Wunnicke, A. L. Roest, et al., "Large redshift in photoluminescence of $p$-doped InP nanowires induced by Fermi-level pinning," Applied Physics Letters, vol. 88, no. 4, Article ID 043109, 3 pages, 2006.

[16] J.-C. Harmand, L. Liu, G. Patriarche, et al., "Potential of semiconductor nanowires for single photon sources," in Quantum Sensing and Nanophotonic Devices, vol. 7222 of Proceedings of SPIE, pp. 1-10, San Jose, Calif, USA, January 2009.

[17] M. Tchernycheva, L. Travers, G. Patriarche, et al., "Au-assisted molecular beam epitaxy of InAs nanowires: growth and theoretical analysis," Journal of Applied Physics, vol. 102, no. 9, Article ID 094313, 2007.

[18] M. Tchernycheva, J.-C. Harmand, G. Patriarche, L. Travers, and G. E. Cirlin, "Temperature conditions for GaAs nanowire formation by Au-assisted molecular beam epitaxy," Nanotechnology, vol. 17, no. 16, pp. 4025-4030, 2006.

[19] T. B. Massalski, Ed., Binary Alloy Phase Diagrams, vol. 1, ASM International, Metals Park, Ohio, USA, 2nd edition, 1990.
[20] J. A. Del Alamo and T. Mizutani, "AuGeNi ohmic contacts to n-InP for FET applications," Solid State Electronics, vol. 31, no. 11, pp. 1635-1639, 1988.

[21] D. G. Ivey, D. Wang, D. Yang, R. Bruce, and G. Knight, "Au/Ge/Ni ohmic contacts to n-Type InP," Journal of Electronic Materials, vol. 23, no. 5, pp. 441-446, 1994.

[22] A. Kolmakov, "The effect of morphology and surface doping on sensitization of quasi-1D metal oxide nanowire gas sensors," in Nanomaterial Synthesis and Integration for Sensors, Electronics, Photonics, and Electro-Optics, vol. 6370 of Proceedings of SPIE, pp. 1-8, Boston, Mass, USA, October 2006.

[23] J. H. He, Y. H. Lin, M. E. McConney, V. V. Tsukruk, Z. L. Wang, and G. Bao, "Enhancing UV photoconductivity of $\mathrm{ZnO}$ nanobelt by polyacrylonitrile functionalization," Journal of Applied Physics, vol. 102, no. 8, Article ID 084303, 2007.

[24] J. D. Prades, R. Jimenez-Diaz, F. Hernandez-Ramirez, et al., "Toward a systematic understanding of photodetectors based on individual metal oxide nanowires," Journal of Physical Chemistry C, vol. 112, no. 37, pp. 14639-14644, 2008.

[25] F. Hernandez-Ramirez, A. Tarancon, O. Casals, et al., "Electrical properties of individual tin oxide nanowires contacted to platinum electrodes," Physical Review B, vol. 76, no. 8, Article ID 085429, 2007.

[26] A. Kolmakov, S. Potluri, A. Barinov, et al., "Spectromicroscopy for addressing the surface and electron transport properties of individual 1-D nanostructures and their networks," ACS Nano, vol. 2, no. 10, pp. 1993-2000, 2008.

[27] S. Dhara, H. S. Solanki, V. Singh, et al., "Magnetotransport properties of individual InAs nanowires," Physical Review B, vol. 79, no. 12, Article ID 121311, 2009.

[28] B. van Zeghbroeck, Principles of Semiconductor Devices and Heterojunctions, Prentice Hall, Englewood Cliffs, NJ, USA, 1st edition, 2008.

[29] M.-H. Ham, J.-H. Choi, W. Hwang, C. Park, W.-Y. Lee, and J.-M. Myoung, "Contact characteristics in GaN nanowire devices,” Nanotechnology, vol. 17, no. 9, pp. 2203-2206, 2006. 

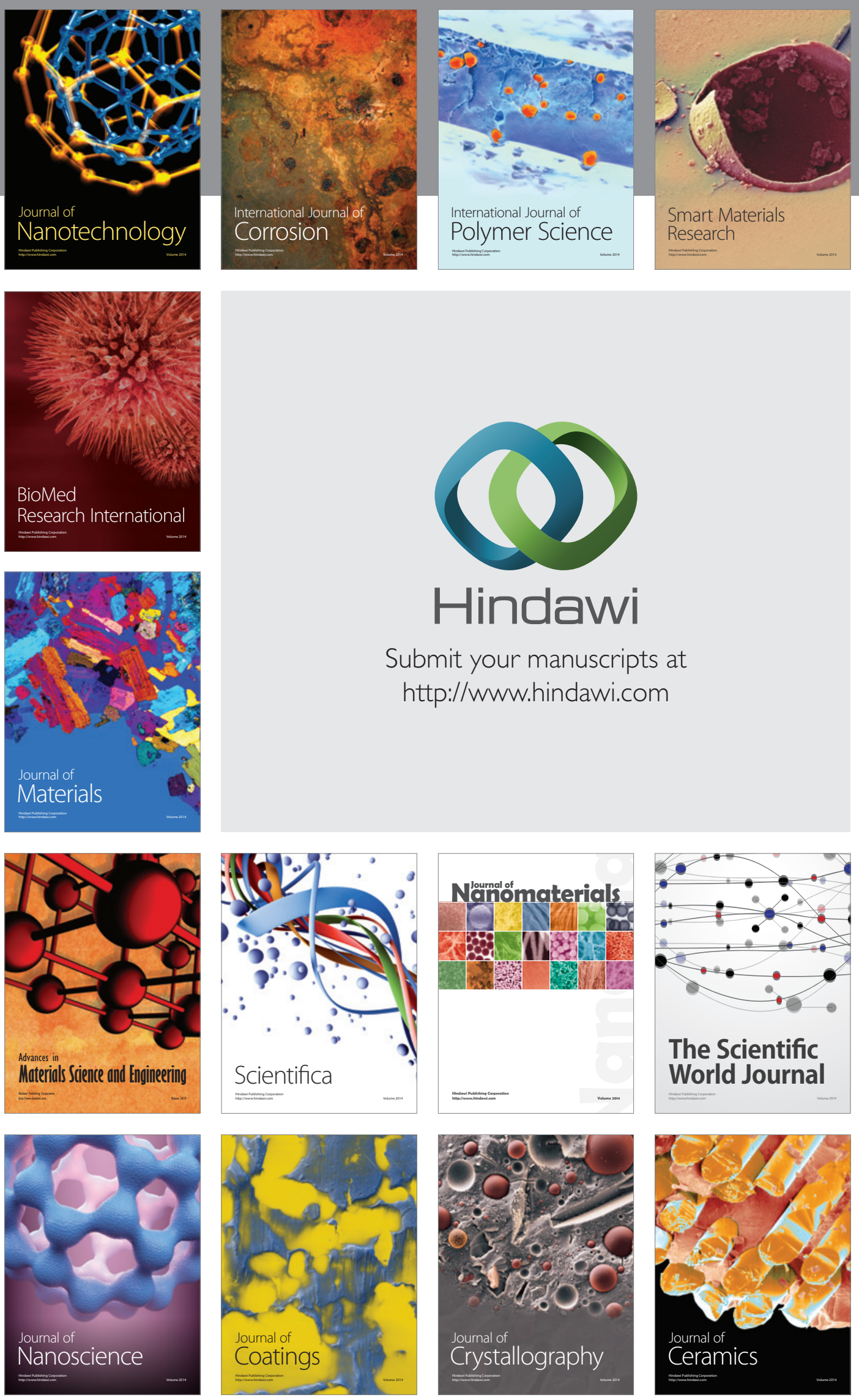

The Scientific World Journal

Submit your manuscripts at

http://www.hindawi.com

\section{World Journal}

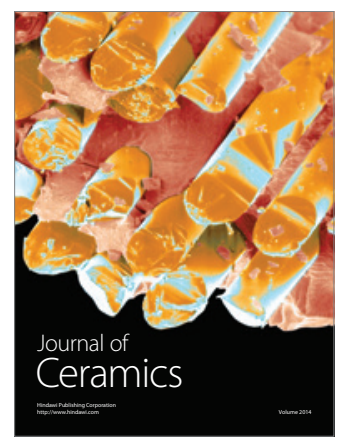

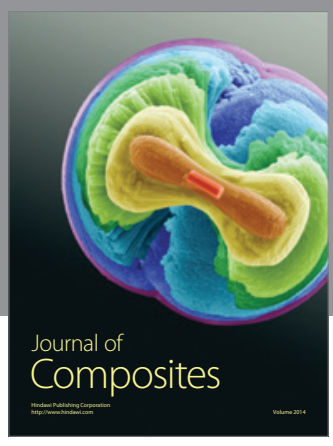
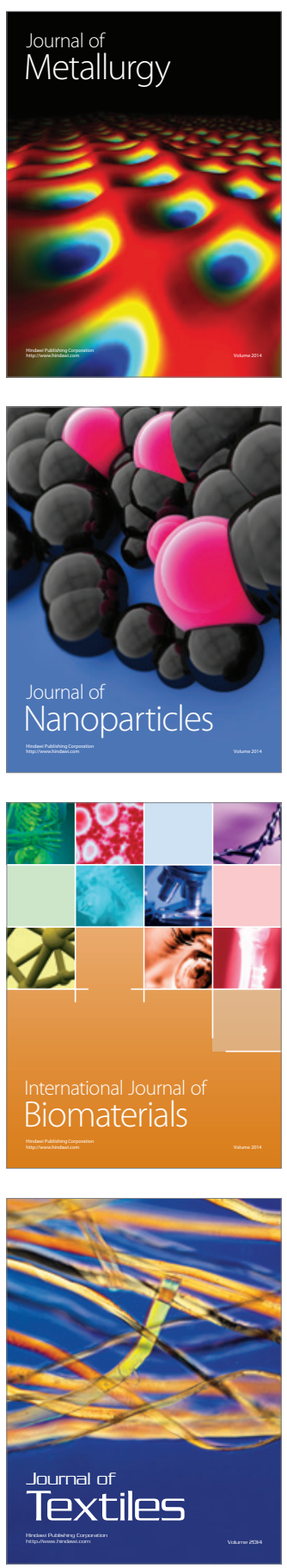